\title{
Expansion of Spherical Bessel Functions in a Series of Chebyshev Polynomials
}

\author{
By A. M. Arthurs and R. McCarroll
}

1. Introduction. In many problems of physics it is necessary to evaluate the spherical Bessel functions over a wide range of argument and up to a high order. For example, their evaluation is necessary in the solution of the integral equations of atomic scattering, as described in the work of Frazer [1].

One method is to generate the functions by means of recurrence formulas [2], [3], [4] which basically provide a large number of orders at a single value of the argument. A method which in essence provides a single order for a given range of argument, and which is suitable for use in automatic computations associated with atomic scattering, is to expand the spherical Bessel functions in terms of Chebyshev polynomials

2. The Method. We introduce the shifted Chebyshev polynomial $T_{n}{ }^{*}(z)$ which satisfies the following differential equation given by Lanczos [5].

$$
\left(z-z^{2}\right) \frac{d^{2} T_{n}^{*}}{d z^{2}}-\frac{(2 z-1)}{2} \frac{d T_{n}^{*}}{d z}+n^{2} T_{n}^{*}=0 \quad 0 \leqq z \leqq 1
$$

The spherical Bessel function

$$
j_{r}(x)=(\pi / 2 x)^{1 / 2} J_{r+1 / 2}(x)
$$

is expanded in series of $T_{n}^{*}(z)$ for $z \leqq 1$ as

$$
j_{r}(x)=(x / 2)^{r} N_{r} \sum_{n} A_{n} T_{n}^{*}(z)
$$

where

$$
z=x^{2} / p
$$

and where $N_{r}$ is the normalization factor given by

$$
N_{r}=\pi^{1 / 2}\left[2 \Gamma(r+3 / 2) \sum_{n}(-1)^{n} A_{n}\right]^{-1},
$$

chosen so that as $x \rightarrow 0$

$$
j_{r}(x) \rightarrow \pi^{1 / 2}(x / 2) r / 2 \Gamma(r+3 / 2) .
$$

The parameter $\boldsymbol{p}$ is chosen according to the required $x$-range.

Substituting the expression (3) into the differential equation satisfied by $j_{r^{\prime}}(x)$, namely,

$$
\left[\frac{d^{2}}{d x^{2}}+\frac{2}{x} \frac{d}{d x}+1-\frac{r(r+1)}{x^{2}}\right] j_{r}(x)=0,
$$

Received June 1, 1960. 
TABLE 1

Tables of Expansion Coefficients $j_{r}(x)=(x / 2) N_{r} \sum_{n} A_{n} T_{n}^{*}\left(x^{2} / 100\right)$ $-10 \leqq x \leqq 10$

\begin{tabular}{|c|c|c|}
\hline n & $j_{0}$ & $\begin{array}{l}\dot{j}_{1} \\
\boldsymbol{A}_{n}\end{array}$ \\
\hline $\begin{array}{r}0 \\
1 \\
2 \\
3 \\
4 \\
5 \\
6 \\
7 \\
8 \\
9 \\
10 \\
11 \\
12 \\
13 \\
14 \\
15\end{array}$ & 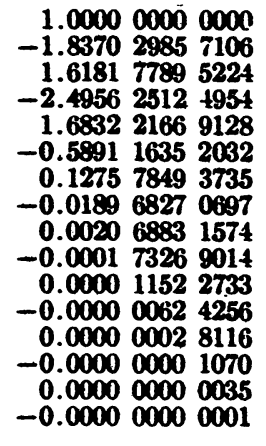 & 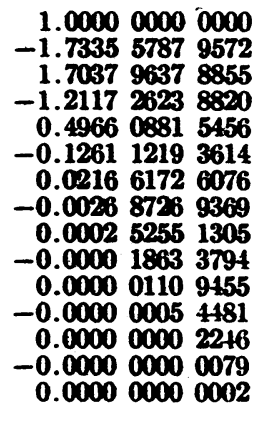 \\
\hline$n$ & $j_{z}$ & $j_{3}$ \\
\hline $\begin{array}{r}0 \\
1 \\
2 \\
3 \\
4 \\
5 \\
6 \\
7 \\
8 \\
9 \\
10 \\
11 \\
12 \\
13\end{array}$ & 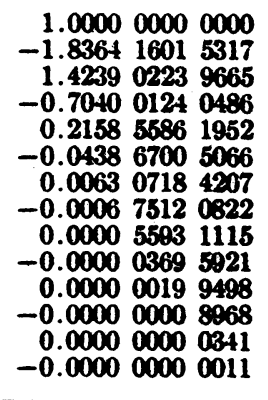 & 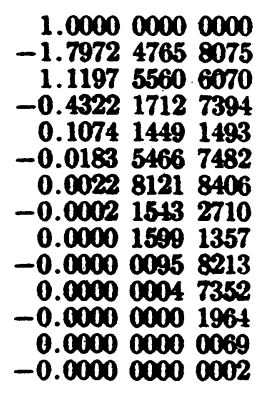 \\
\hline$n$ & $\begin{array}{l}j_{1} \\
A_{n}\end{array}$ & $\boldsymbol{A}_{\mathbf{n}}$ \\
\hline $\begin{array}{r}0 \\
1 \\
2 \\
3 \\
4 \\
5 \\
6 \\
7 \\
8 \\
9 \\
10 \\
11 \\
12\end{array}$ & 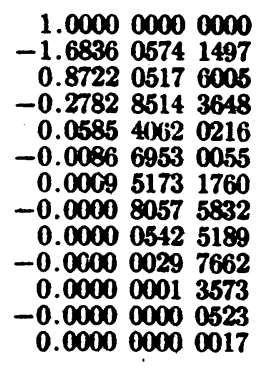 & 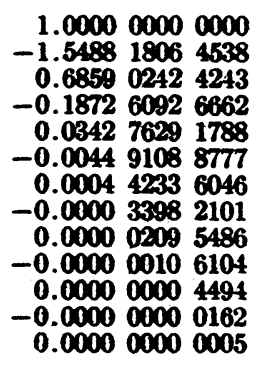 \\
\hline * & $\dot{A}_{\boldsymbol{n}}$ & ${ }_{i}$ \\
\hline $\begin{array}{r}0 \\
1 \\
2 \\
3 \\
4 \\
5 \\
6 \\
7 \\
8 \\
9 \\
10 \\
11 \\
12\end{array}$ & $\begin{array}{rrr}1.0000 & 0000 & 0000 \\
-1.4171 & 3242 & 2748 \\
0.5479 & 5705 & 4665 \\
-0.1310 & 4234 & 0105 \\
0.0212 & 7626 & 4218 \\
-0.0025 & 0394 & 3447 \\
0.0002 & 2390 & 8476 \\
-0.0000 & 1575 & 8616 \\
0.0000 & 0089 & 6941 \\
-0.0000 & 0004 & 2186 \\
0.0000 & 0000 & 1669 \\
-0.0000 & 0000 & 0056 \\
0.0000 & 0000 & 0002\end{array}$ & 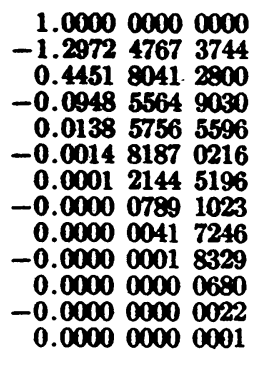 \\
\hline
\end{tabular}


Tables of Expansion Coefficients-Continued

\begin{tabular}{|c|c|c|}
\hline$n$ & $\begin{array}{l}j_{2} \\
A_{n}\end{array}$ & $\dot{j}_{n}$ \\
\hline $\begin{array}{r}0 \\
1 \\
2 \\
3 \\
4 \\
5 \\
6 \\
7 \\
8 \\
9 \\
10 \\
11\end{array}$ & $\begin{array}{rrr}1.0000 & 0000 & 0000 \\
-1.1911 & 3494 & 9922 \\
0.3675 & 0203 & 3005 \\
-0.0706 & 7640 & 7499 \\
0.0093 & 9388 & 4497 \\
-0.0009 & 2119 & 2206 \\
0.0000 & 6972 & 0100 \\
-0.0000 & 0420 & 9178 \\
0.0000 & 0020 & 7889 \\
-0.0000 & 0000 & 8569 \\
0.0000 & 0000 & 0300 \\
-0.0000 & 0000 & 0009\end{array}$ & $\begin{array}{rrr}1.0000 & 0000 & 0000 \\
-1.0981 & 6702 & 5095 \\
0.3078 & 0618 & 7533 \\
-0.0539 & 7859 & 5631 \\
0.0065 & 8545 & 8180 \\
-0.0005 & 9665 & 6926 \\
0.0000 & 4196 & 8280 \\
-0.0000 & 0236 & 6972 \\
0.0000 & 0010 & 9704 \\
-0.0000 & 0000 & 4260 \\
0.0000 & 0000 & 0141 \\
-0.0000 & 0000 & 0004\end{array}$ \\
\hline$n$ & $i_{\Lambda_{n}}$ & $j_{A_{n}}^{j_{n}}$ \\
\hline $\begin{array}{r}0 \\
1 \\
2 \\
3 \\
4 \\
5 \\
6 \\
7 \\
8 \\
9 \\
10 \\
11\end{array}$ & $\begin{array}{rrr}1.0000 & 0000 & 0000 \\
-1.0168 & 7201 & 6138 \\
0.2611 & 5956 & 8765 \\
-0.0421 & 0983 & 2180 \\
0.0047 & 5003 & 6264 \\
-0.0004 & 0010 & 7956 \\
0.0000 & 2629 & 5870 \\
-0.0000 & 0139 & 1880 \\
0.0000 & 0006 & 0782 \\
-0.0000 & 0000 & 2232 \\
0.0000 & 0000 & 0070 \\
-0.0000 & 0000 & 0002\end{array}$ & $\begin{array}{rrr}1.0000 & 0000 & 0000 \\
-0.9456 & 3737 & 0019 \\
0.2241 & 3285 & 9656 \\
-0.0334 & 5827 & 7279 \\
0.0035 & 1079 & 6899 \\
-0.0002 & 7638 & 9359 \\
0.0000 & 1705 & 0581 \\
-0.0000 & 0085 & 0428 \\
0.0000 & 0003 & 5115 \\
-0.0000 & 0000 & 1223 \\
0.0000 & 0000 & 0036 \\
-0.0000 & 0000 & 0001\end{array}$ \\
\hline$n$ & $j_{A_{n}}^{j_{12}}$ & $j_{12}^{j_{12}}$ \\
\hline $\begin{array}{r}0 \\
1 \\
2 \\
3 \\
4 \\
5 \\
6 \\
7 \\
8 \\
9 \\
10\end{array}$ & $\begin{array}{rrr}1.0000 & 0000 & 0000 \\
-0.8829 & 7010 & 1098 \\
0.1943 & 1493 & 5452 \\
-0.0270 & 1121 & 0330 \\
0.0026 & 5020 & 1752 \\
-0.0001 & 9588 & 4596 \\
0.0000 & 1138 & 8223 \\
-0.0000 & 0053 & 7112 \\
0.0000 & 0002 & 1036 \\
-0.0000 & 0000 & 0697 \\
0.0000 & 0000 & 0020\end{array}$ & $\begin{array}{rrr}1.0000 & 0000 & 0000 \\
-0.8275 & 7427 & 4817 \\
0.1699 & 8535 & 0546 \\
-0.0221 & 1284 & 8562 \\
0.0020 & 3772 & 5111 \\
-0.0001 & 4196 & 6316 \\
0.0000 & 0780 & 5415 \\
-0.0000 & 0034 & 9193 \\
0.0000 & 0001 & 3008 \\
-0.0000 & 0000 & 0411 \\
0.0000 & 0000 & 0011\end{array}$ \\
\hline$n$ & $j_{A_{n}}^{j_{11}}$ & $\begin{array}{l}j_{1 s} \\
A_{n}\end{array}$ \\
\hline $\begin{array}{r}0 \\
1 \\
2 \\
3 \\
4 \\
5 \\
6 \\
7 \\
8 \\
9 \\
10\end{array}$ & $\begin{array}{rrr}1.0000 & 0000 & 0000 \\
-0.7783 & 5629 & 2813 \\
0.1498 & 9703 & 5766 \\
-0.0183 & 2686 & 5868 \\
0.0015 & 9235 & 3331 \\
-0.0001 & 0493 & 0348 \\
0.0000 & 0547 & 2793 \\
-0.0000 & 0023 & 2885 \\
0.0000 & 0000 & 8272 \\
-0.0000 & 0000 & 0250 \\
0.0000 & 0000 & 0006\end{array}$ & $\begin{array}{rrr}1.0000 & 0000 & 0000 \\
-0.7344 & 0438 & 9543 \\
0.1331 & 3186 & 9771 \\
-0.0153 & 5579 & 5806 \\
0.0012 & 6227 & 9463 \\
-0.0000 & 7891 & 7018 \\
0.0000 & 0391 & 5367 \\
-0.0000 & 0015 & 8871 \\
0.0000 & 0000 & 5393 \\
-0.0000 & 0000 & 0156 \\
0.0000 & 0000 & 0004\end{array}$ \\
\hline
\end{tabular}


TABLE 2

Table of Normalization Factors

\begin{tabular}{|c|c|}
\hline$r$ & $v_{r}$ \\
\hline $\begin{array}{r}0 \\
1 \\
2 \\
3 \\
4 \\
5 \\
6 \\
7 \\
8 \\
9 \\
10 \\
11 \\
12 \\
13 \\
14 \\
15\end{array}$ & $\begin{array}{cccc}10^{-1} \cdot 1.0670 & 1130 & 3957 \\
10^{-1} \cdot 1.0588 & 0224 & 7273 \\
10^{-2} \cdot 5.0977 & 3196 & 1602 \\
10^{-2} \cdot 1.7016 & 2862 & 5983 \\
10^{-3} \cdot 4.3387 & 2970 & 2098 \\
10^{-4} \cdot 8.8939 & 6388 & 4585 \\
10^{-4} \cdot 1.5178 & 7598 & 0079 \\
10^{-6} \cdot 2.2135 & 3657 & 5215 \\
10^{-6} \cdot 2.8143 & 4241 & 8551 \\
10^{-7} \cdot 3.1696 & 2427 & 4560 \\
10^{-8} \cdot 3.2028 & 4879 & 3012 \\
10^{-9} \cdot 2.9343 & 5222 & 5384 \\
10^{-10} \cdot 2.4587 & 5268 & 1163 \\
10^{-11} \cdot 1.8981 & 3134 & 3689 \\
10^{-12} \cdot 1.3584 & 9275 & 4731 \\
10^{-14} \cdot 9.0623 & 7664 & 9006\end{array}$ \\
\hline
\end{tabular}

and using the properties of the shifted Chebyshev polynomials, we obtain a set of simultaneous linear algebraic equations which may be solved for the ratios $A_{1} / A_{0}$, $A_{2} / A_{0}, \cdots$. The solutions may be normalized, using the factors given by (5).

3. Calculations and Results. The calculation of the coefficients $A_{n}$ has been carried out on a DEUCE digital computer for $p=100$, and $r=0$ to 15 . For all values of $r, A_{0}$ has been chosen as 1.0 , and the ratios $A_{n} / A_{0}$ computed accordingly. Tables of the coefficients, together with the corresponding normalization factors are presented herein. The expansion coefficients are given to 12 decimal places to insure that for the range of $x$ considered the spherical Bessel functions should be accurate to 10 significant figures.

As can be seen from the tables, the convergence of the coefficients is very rapid; if the Chebyshev expansion and Taylor series are curtailed after $n$ terms, the ratio of the $(n+1)$ th terms is about $1 / 2^{n-1}$.

The authors wish to thank Mrs. N. Scott and Miss M. Wilson for assistance with the computations.

The Queen's University of Belfast

N. Ireland

Mathematical Institute

Oxford, England

1. P. A. Frazer, Sci. Rep., Univ. Western Ontario, No. 4, 1958.

2. I. A. Stegun \& M. Abramowitz, "Generation of Bessel functions on high speed computers," MTAC, v. 11, 1957, 255-257.

3. F. J. Corbató \& J. L. Uretsky, "Generation of spherical Bessel functions in digital computers," J. Assoc. Comput. Mach., v. 6, 1959, p. 366-375.

4. M. Goldstein \& R. M. Thater, "Recurrence techniques for the calculation of Bessel functions," $M T A C$, v. 13, 1959, p. 102-108.

5. C. Lanczos, "Tables of Chebyshev polynomials, $S_{n}(x)$ and $C_{n}(x)$," Nat. Bur. Standards, Appl. Math. Ser. No. 9, U. S. Government Printing Office, Washington, I). C., 1952. 